\title{
Rapid detection of genetic mutations in individual breast cancer patients by next-generation DNA sequencing
}

\author{
Suqin Liu', Hongjiang Wang ${ }^{1}$, Lizhi Zhang ${ }^{1}$, Chuanning Tang ${ }^{2}$, Lindsey Jones ${ }^{3}$, Hua Ye ${ }^{2}$, Liying Ban ${ }^{1}$, Aman Wang ${ }^{1}$, \\ Zhiyuan Liư', Feng Lou'2, Dandan Zhang ${ }^{2}$, Hong Sun², Haichao Dong ${ }^{2}$, Guangchun Zhang ${ }^{2}$, Zhishou Dong ${ }^{2}$, \\ Baishuai Guo ${ }^{2}$, He Yan², Chaowei Yan², Lu Wang ${ }^{2}$, Ziyi Su², Yangyang Li ${ }^{2}$, Xue F Huang ${ }^{3}$, Si-Yi Chen ${ }^{3 *}$ and Tao Zhou ${ }^{\text {* }}$
}

\begin{abstract}
Breast cancer is the most common malignancy in women and the leading cause of cancer deaths in women worldwide. Breast cancers are heterogenous and exist in many different subtypes (luminal A, luminal B, triple negative, and human epidermal growth factor receptor 2 (HER2) overexpressing), and each subtype displays distinct characteristics, responses to treatment, and patient outcomes. In addition to varying immunohistochemical properties, each subtype contains a distinct gene mutation profile which has yet to be fully defined. Patient treatment is currently guided by hormone receptor status and HER2 expression, but accumulating evidence suggests that genetic mutations also influence drug responses and patient survival. Thus, identifying the unique gene mutation pattern in each breast cancer subtype will further improve personalized treatment and outcomes for breast cancer patients. In this study, we used the lon Personal Genome Machine (PGM) and Ion Torrent AmpliSeq Cancer Panel to sequence 737 mutational hotspot regions from 45 cancer-related genes to identify genetic mutations in 80 breast cancer samples of various subtypes from Chinese patients. Analysis revealed frequent missense and combination mutations in PIK3CA and TP53, infrequent mutations in PTEN, and uncommon combination mutations in luminal-type cancers in other genes including BRAF, GNAS, IDH1, and KRAS. This study demonstrates the feasibility of using lon Torrent sequencing technology to reliably detect gene mutations in a clinical setting in order to guide personalized drug treatments or combination therapies to ultimately target individual, breast cancer-specific mutations.
\end{abstract}

Keywords: Breast cancer, Genetic mutations, lon torrent sequencing, Targeted therapy, Personalized medicine

\section{Introduction}

Breast cancer is the second most common malignancy worldwide and the most frequent in women. Roughly 1.67 million new cases and 522,000 deaths were reported globally in 2012, making breast cancer the fifth leading cause of cancer death. Breast cancer incidence differs with population and geographic location, where China alone accounted for more than 187,000 cases and nearly 48,000 deaths in 2012, whereas over 230,000 cases and more than 43,000 deaths were reported in the US [1].

\footnotetext{
* Correspondence: zhoutao1967@163.com; siyichen@usc.edu

${ }^{1}$ The First Affiliated Hospital of Dalian Medical University, Dalian, Liaoning, China

${ }^{3}$ Norris Comprehensive Cancer Center, Department of Molecular Microbiology and Immunology, Keck School of Medicine, University of Southern California, Los Angeles, CA, USA

Full list of author information is available at the end of the article
}

Patient screening is superior in the US than in China [2], which may account for a higher incidence despite a much smaller population. While risk factors for developing breast cancer include ethnicity, older age, and environmental factors, lifestyle and diet also play a significant role, where westernization in Asia is thought to have contributed to the rise in spontaneous breast cancer incidence in Chinese populations over the last 20 years [3-5].

Breast cancers are highly heterogenous and may display different characteristics of hormone receptors (HR) (estrogen receptor (ER) and progesterone receptor (PR)) and human epidermal growth factor receptor 2 (HER2) status, and together this information helps to distinguish different types of breast cancers: luminal A (HR+/HER2-, tumor grade 1 or 2$)$, luminal B/HER2(HR+/HER2-, tumor grade 3 or 4$)$, luminal B/HER2+ 
$(\mathrm{HR}+/ \mathrm{HER} 2+)$, triple negative (HR-/HER2-), or HER2 overexpressing (HR-/HER2+) [6]. Together luminal A and $B$ subtypes account for $65 \%-70 \%$ of all breast cancers, whereas $10 \%-15 \%$ are triple negative and $10 \%-$ $20 \%$ are HER2 overexpressing [7].

These distinct types of breast cancers all have different characteristics, behaviors, and prognoses and also respond differently to drug treatments. Nearly three quarters of all breast cancers are ER+ and are therefore in some way dependent on estrogen for growth, providing a useful target for treating these cancers via ER modulators or downregulators or aromatase inhibitors [8]. But only $20 \%-40 \%$ of patients with advanced ER+ breast cancer have a response to endocrine therapy, which only averages 8 to 14 months [9]. Luminal A types tend to have the best outcome with a 95\% 5-year survival rate, whereas luminal B tumors, which tend to have lower HR expression and subsequently less sensitivity to endocrine therapy but increased sensitive to chemotherapy, tend to have a worse outcome [10-12]. Typically found in younger patients, triple-negative breast cancers (TNBCs) are known to be particularly aggressive and are associated with germline $B R C A$ mutations. TNBCs also have higher relapse rates and decreased overall patient survival than other breast cancer types [13]. HER2-overexpressing breast cancers also have poor prognoses and high metastases rates, and as they lack HR expression, they do not respond to endocrine therapies and are resistant to current chemotherapies [14]. These distinct breast cancer types can be further divided into a multitude of subtypes, and all of these types and subtypes exhibit distinct gene mutation patterns that have yet to be fully defined [10].

Currently, patient prognoses and treatment regimens for breast cancer are guided by the aforementioned characteristics of the tumor, but accumulating evidence suggests that this information is not enough; risk assessments, treatments, and patient outcomes are also influenced by both germline and somatic gene mutations. Known genetic factors like inherited $B R C A$ mutations confer a lifetime risk of developing breast cancer of $60 \%$ to $85 \%$; however, these mutations account for only $2 \%-3 \%$ of all breast cancer cases [15]. Spontaneous mutations in PIK3CA are a much more common event in breast cancers, with more than a quarter of breast cancer patients harboring a mutation in this gene $[16,17]$. While PIK3CA mutations have been shown to be associated with improved patient prognoses, these genetic aberrations have also been shown to impart resistance to trastuzumab, a common treatment option for HER2-overexpressing breast cancers [18]. Identifying gene mutations in patients with TNBC is especially important because these cancers currently do not have direct targets for treatments. Therefore, it is important to establish both the immunohistochemical properties and genetic profile of each breast cancer tumor to optimize treatment regimens and avoid unnecessary drug toxicities and ultimately to improve patient outcomes.

There are a number of different next-generation sequencing (NGS) platforms available, including Illumina, 454, and SOLiD, but these are typically expensive both in instrument and assay cost, and therefore, these tools are unrealistic for widespread clinical diagnostic use. But new technology like the Ion Torrent sequencing platform has been shown to be more cost and time effective with reliable results [19], which may help make cancer DNA sequencing and personalized treatments a reality for each cancer patient in the near future. In the present study, we have used Ion Torrent sequencing technology with the Ion Personal Genome Machine (PGM) and Ion Torrent AmpliSeq Cancer Panel as a rapid and affordable method to detect gene mutations in 80 clinical breast cancer samples of different types from Chinese patients.

\section{Materials and methods \\ Ethics statement}

The study has been approved by the Human Research Ethics Committee of the First Affiliated Hospital of Dalian Medical University, China. The institutional ethics committee waived the need for consent for formalin-fixed, paraffin-embedded (FFPE) tumor samples obtained from the tumor tissue bank at the hospital's Department of Pathology. All samples and medical data used in this study have been irreversibly anonymized.

\section{Patient information}

Tumor samples used in the study were collected from the First Affiliated Hospital of Dalian Medical University, China. A total of 80 FFPE tumor samples from Chinese breast cancer patients were analyzed (Table 1). Patients were an average of 55 years old, with a range of 30-75 years. American Joint Committee on Cancer (AJCC)/ tumor, node, and metastasis (TNM) cancer staging was assessed, and tumor samples were also analyzed for immunohistochemical status of HR and HER2 (Table 1). Based on these, patients were categorized into five breast cancer subtypes: luminal A (HR+/HER2-, AJCC stage 1 or 2; 26.3\%), luminal B/HER2- (HR+/HER2-, AJCC stage 3 or $4 ; 21.3 \%$ ), luminal B/HER2+ (HR+/HER2+; $30.0 \%$ ), triple negative (HR-/HER2-; 7.5\%), and HER2 overexpressing (HR-/HER $2+; 8.8 \%$ ), and five samples (6.3\%) were unclassifiable due to unknown HER2 status (Table 2).

\section{DNA preparation}

FFPE tissue samples were first deparaffinized in xylene, $3-5-\mu \mathrm{m}$-thick sections were extracted, and DNA was 
Table 1 Clinical features of 80 breast cancer patients

\begin{tabular}{|c|c|c|}
\hline Characteristic & & $n(\%)$ \\
\hline \multirow[t]{2}{*}{ Age (years) } & Median: 56 & \\
\hline & Range: 30-75 & \\
\hline \multirow[t]{2}{*}{ HR status } & $\mathrm{HR}+$ & $65(81.3 \%)$ \\
\hline & $\mathrm{HR}-$ & $15(18.8 \%)$ \\
\hline \multirow[t]{5}{*}{ HER2 status } & HER2+ & $12(15.0 \%)$ \\
\hline & HER2++ & $18(22.5 \%)$ \\
\hline & HER2+++ & $1(1.3 \%)$ \\
\hline & HER2- & $44(55.0 \%)$ \\
\hline & Unknown & $5(6.3 \%)$ \\
\hline \multirow[t]{6}{*}{ AJCC/TNM stage } & $2 a$ & 19 (23.8\%) \\
\hline & $2 b$ & $26(32.5 \%)$ \\
\hline & $3 a$ & $23(28.8 \%)$ \\
\hline & $3 b$ & $4(8.8 \%)$ \\
\hline & $3 c$ & 7 (8.8\%) \\
\hline & 4 & $1(1.3 \%)$ \\
\hline \multirow{3}{*}{$\begin{array}{l}\text { Pathological diagnosis } \\
\text { of infiltrating ductal } \\
\text { carcinoma }\end{array}$} & IDC2 & $71(88.8 \%)$ \\
\hline & IDC3 & $6(7.5 \%)$ \\
\hline & Others & $3(3.8 \%)$ \\
\hline
\end{tabular}

isolated using the QIAamp DNA Mini Kit (QIAGEN) as per the manufacturer's instructions.

Ion Torrent PGM library preparation and DNA sequencing An Ion Torrent adapter-ligated library was constructed with the Ion AmpliSeq Library Kit 2.0 (Life Technologies, Part \#4475345 Rev. A) following the manufacturer's protocol. Briefly, $50 \mathrm{ng}$ of pooled amplicons were end- repaired, Ion Torrent adapters P1 and A were ligated, and the adapter-ligated products were then purified with AMPure beads (Beckman Coulter, Brea, CA, USA), nicktranslated, and PCR-amplified for 5 cycles. The resulting library was purified with AMPure beads (Beckman Coulter), and the library concentration and size was determined with the Agilent 2100 Bioanalyzer and Agilent Bioanalyzer DNA High-Sensitivity LabChip (Agilent Technologies).

Sample emulsion PCR, emulsion breaking, and enrichment were performed with the Ion PGM 200 Xpress Template Kit (Life Technologies, Part \#4474280 Rev. B), according to the manufacturer's instructions. Briefly, an input concentration of one DNA template copy/Ion Sphere Particles (ISPs) was added to emulsion PCR master mix, and the emulsion was generated with an IKADT-20 mixer (Life Technologies). Next, ISPs were recovered, and template-positive ISPs were enriched with Dynabeads MyOne Streptavidin C1 beads (Life Technologies). The Qubit 2.0 fluorometer (Life Technologies) was used to confirm ISP enrichment. Threehundred sixteen chips were used to sequence barcoded samples on the Ion Torrent PGM for 65 cycles, and an Ion PGM 200 Sequencing Kit (Life Technologies, Part \# 4474004 Rev. B) was used for sequencing reactions, as per the recommended protocol.

This Personalized Cancer Mutation Panel targets 737 mutational hotspot regions in the following 45 genes: $A B L 1, A K T 1, A L K, A P C, A T M, B R A F, C D H 1, C D K N 2 A$, CSF1R, CTNNB1, EGFR, ERBB2, ERBB4, FBXW7, FGFR1, FGFR2, FGFR3, FLT3, GNAS, HNF1A, HRAS, IDH1, JAK3, KDR, KIT, KRAS, MET, MLH1, MPL, NOTCH1, NPM1, NRAS, PDGFRA, PIK3CA, PTEN,

Table 2 Average patient age, average disease-free survival (DFS), and mutation frequency in breast cancer subtypes with or without mutations

\begin{tabular}{|c|c|c|c|c|c|c|c|c|c|c|c|}
\hline Subtype & $\begin{array}{l}n(\% \\
\text { total) }\end{array}$ & $\begin{array}{l}\text { Average } \\
\text { age } \\
\text { (years) }\end{array}$ & $\begin{array}{l}\text { Average } \\
\text { DFS } \\
\text { (months) }\end{array}$ & $\begin{array}{l}\text { Samples } \\
\text { with } \\
\text { mutations } \\
\text { (freq.) }\end{array}$ & $\begin{array}{l}\text { Samples } \\
\text { without } \\
\text { mutations } \\
\text { (freq.) }\end{array}$ & $\begin{array}{l}\text { Average } \\
\text { age with } \\
\text { mutations } \\
\text { (years) }\end{array}$ & $\begin{array}{l}\text { Average age } \\
\text { without } \\
\text { mutations } \\
\text { (months) }\end{array}$ & $\begin{array}{l}P \\
\text { value }\end{array}$ & $\begin{array}{l}\text { Average } \\
\text { DFS with } \\
\text { mutations } \\
\text { (months) }\end{array}$ & $\begin{array}{l}\text { Average DFS } \\
\text { without } \\
\text { mutations } \\
\text { (months) }\end{array}$ & $\begin{array}{l}P \\
\text { value }\end{array}$ \\
\hline All & $\begin{array}{l}80 \\
(100 \%)\end{array}$ & 54.5 & 38.3 & 32 (40.0\%) & 48 (60.0\%) & 54.9 & 54.2 & 0.757 & 38.8 & 38.0 & 0.741 \\
\hline Luminal A & $\begin{array}{l}21 \\
(26.3 \%)\end{array}$ & 53.7 & 39.0 & $9(42.9 \%)$ & $12(57.1 \%)$ & 53.8 & 53.7 & 0.979 & 40.6 & 37.8 & 0.407 \\
\hline $\begin{array}{l}\text { Luminal B/ } \\
\text { HER2-- }\end{array}$ & $\begin{array}{l}17 \\
(21.3 \%)\end{array}$ & 55.8 & 39.4 & $6(35.3 \%)$ & $11(64.7 \%)$ & 57.3 & 54.9 & 0.626 & 40.5 & 38.7 & 0.799 \\
\hline $\begin{array}{l}\text { Luminal B/ } \\
\text { HER2+ }\end{array}$ & $\begin{array}{l}24 \\
(30.0 \%)\end{array}$ & 52.9 & 37.3 & $11(45.8 \%)$ & $13(54.2 \%)$ & 53.6 & 52.3 & 0.772 & 37.5 & 37.1 & 0.936 \\
\hline Triple negative & $\begin{array}{l}6 \\
(7.5 \%)\end{array}$ & 53.8 & 37.5 & $4(66.7 \%)$ & $2(33.3 \%)$ & 58.8 & 44.0 & 0.165 & 39.8 & 33.0 & 0.508 \\
\hline $\begin{array}{l}\text { HER2 } \\
\text { overexpressing }\end{array}$ & $\begin{array}{l}7 \\
(8.8 \%)\end{array}$ & 56.6 & 34.6 & $2(28.6 \%)$ & $5(71.4 \%)$ & 52.5 & 58.2 & 0.633 & 31.5 & 35.8 & 0.595 \\
\hline Unknown & $\begin{array}{l}5 \\
(6.3 \%)\end{array}$ & 59.0 & 43.0 & $0(0.0 \%)$ & $5(100 \%)$ & - & 59.0 & - & - & 43.0 & - \\
\hline
\end{tabular}


PTPN11, RB1, RET, SMAD4, SMARCB1, SMO, SRC, STK11, TP53, and VHL.

\section{Variant calling}

Initial data from the PGM runs were processed with the Ion Torrent platform-specific pipeline software Torrent Suite to generate sequence reads, then trim adapter sequences, filter, and remove poor signalprofile reads. Torrent Suite software v3.4 with a plug-in "variant caller v3.4" program was used to generate initial variant calling from the Ion AmpliSeq sequencing data. In order to eliminate erroneous base calling and generate final variant calling, several filtering steps were used: defining average total coverage depth, variant coverage, variant frequency of each sample, and $P$ value $<0.01$; visually inspecting and removing DNA strand-specific errors; defining variants within hotspots; and eliminating variants in amplicon AMPL339432 (PIK3CA, exon13, chr3:178938822-178938906) which is not uniquely matched in the human genome.

\section{Sequence coverage}

From the 80 samples, the mean read length was $76 \mathrm{bp}$ and the average reads were approximately $24 \mathrm{Mb}$ of sequence per sample. With normalization to 300,000 reads per specimen, there was an average of 1,639 reads per amplicon (range: 22 to 6,020) (Figure 1A); 180/189 (95.2\%) amplicons averaged at least 100 reads; and 170/ $189(89.9 \%)$ amplicons averaged at least 300 reads (Figure 1B).

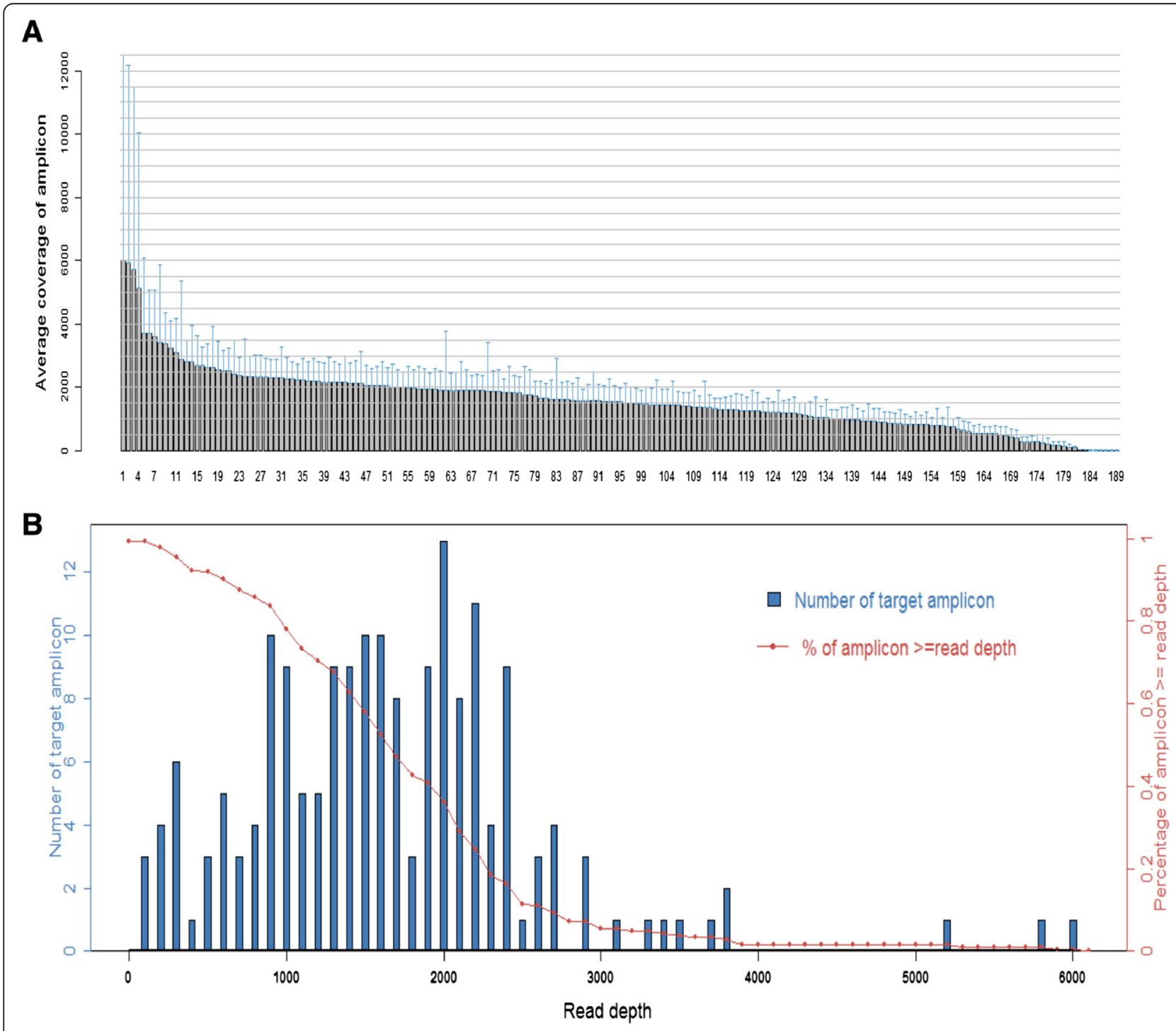

Figure 1 Sequence read distribution across 189 amplicons generated from 80 breast cancer samples, normalized to 300,000 reads per sample. (A) Average number of reads observed for each amplicon. (B) Number of targets with a given read depth, sorted in bins of 100 reads. 


\section{Somatic mutations}

Detected mutations were compared to variants in the 1000 Genomes Project [20] and 6,500 exomes of the National Heart, Lung, and Blood Institute Exome Sequencing Project [21] to distinguish between somatic and germline mutations.

\section{Bioinformatical and experimental validation}

We used the COSMIC [22] (version 64), MyCancerGenome database (http://www.mycancergenome.org/), and some publications to assess recurrent mutations in breast cancer (Additional file 1: Table S1). Additionally, detected missense mutations were confirmed by Sanger sequencing (data not shown). All mutations identified with Sanger sequencing were consistent with those identified with the Ion Torrent PGM.

\section{Statistical analysis}

The Fisher's exact test was used to define significant values in the detected mutated genes, and the total variants and odds ratios (OR) between samples with mutations and without mutations were determined using $2 \times$ 2 contingency tables and the GraphPad QuickCalcs online calculator for Scientists (http://www.graphpad.com/ quickcalcs/index.cfm). All $P$ values are two-sided, and statistical significance was defined as $P<0.05$.

\section{Results and discussion}

From the 45 genes screened in our study, 39 mutations were detected in 32 of 80 samples (40.0\%) (Figure 2). Except for the five unclassified samples with no mutations, mutations were detected at different frequencies across all breast cancer subtypes (Table 2). Triple negative samples contained the highest mutation frequency (66.7\%), whereas HER2-overexpressing samples contained the lowest mutation frequency (28.6\%), and luminal A, luminal B/HER2-, and luminal B/HER2+ had similar mutation frequencies $(42.9 \%, 35.3 \%$, and $45.8 \%$, respectively). Twenty-six samples (32.5\%) contained one mutation, five samples $(6.3 \%)$ contained two mutations, and one sample (1.3\%) contained three mutations, and interestingly, combination mutations were only found in the luminal subtypes (Table 3). PIK3CA mutations and TP53 mutations were the most prevalent $(32.5 \%$ and $10.0 \%$, respectively), and mutations were also identified in BRAF, GNAS, IDH1, KRAS, and PTEN all at a frequency of $1.3 \%$ (Figure 3 ). Among each subtype, there was no statistically significant difference in age or disease-free survival (DFS) between patients with mutations and patients without mutations (Table 2).

\section{PIK3CA mutations}

Twenty-six samples (32.5\%) harbored PIK3CA mutations, which accounted for $66.7 \%$ of all detected mutations in our study. Five different PIK3CA mutations were identified: p.N345K in the $\mathrm{C} 2$ domain encoded by exon 4, p.E542K and p.E545K in the helical domain encoded by exon 9, and p.H1047R and p.H1047L in the kinase domain encoded by exon 20. Mutations p.E542K, p.E545K, and p.H1047R have been found in previous studies to be the most prevalent in human breast cancers and are associated with an increase in kinase activity in the

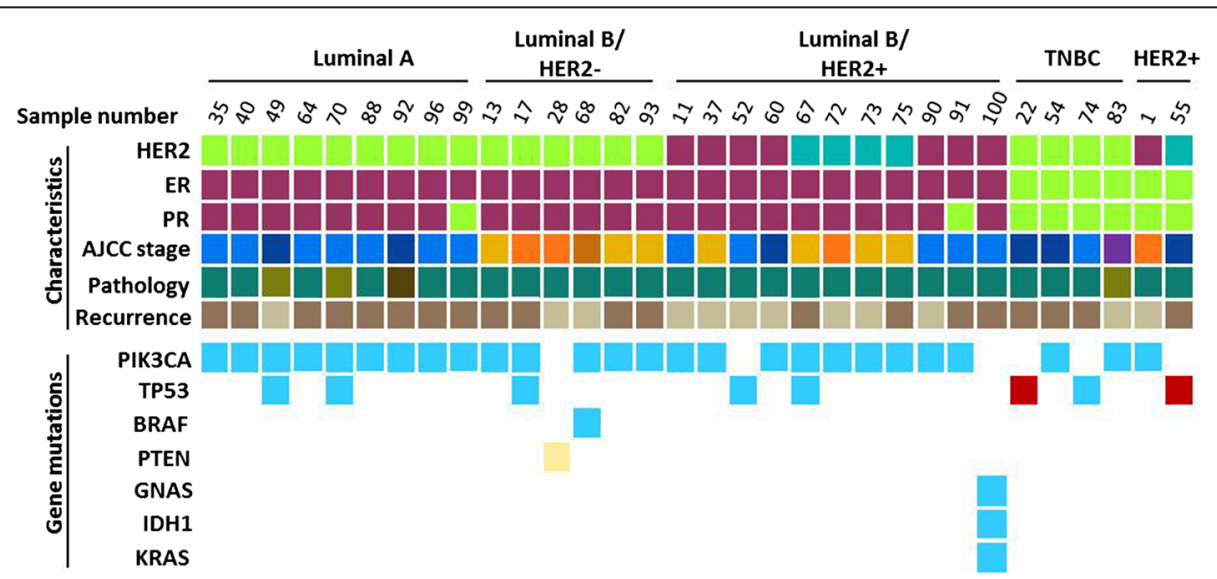

\begin{tabular}{|c|c|c|c|c|}
\hline HR status & AJCC stage & Pathology & Recurrence & Mutation type \\
\hline negative & $2 a$ & IDC2 & recurrence & missense \\
\hline positive & $2 b$ & IDC3 & no recurrence & nonsense \\
\hline high positive & $3 a$ & other & & deletion \\
\hline
\end{tabular}

Figure $\mathbf{2}$ Summary of mutated genes detected in $\mathbf{8 0}$ breast cancer samples. Thirty-two samples harbor mutations in PIK3CA, TP53, KRAS, BRAF, PTEN, GNAS, and IDH1. Samples are classified by four methods: 1) Immunohistochemistry of ER, PR, and HER2; 2) pathologic type (IDC2, IDC3, other); 3) AJCC/TNM-staging (2a, 2b, 3a, 3b, 3c, 4); and 4) recurrence or no recurrence. 
Table 3 Detected point mutations per breast cancer subtype

\begin{tabular}{|c|c|c|c|c|c|c|c|c|c|}
\hline Breast cancer type & Gene(s) & Mutation(s) & Age (years) & DFS (months) & Recurrence & AJCC & ER & PR & HER2 \\
\hline \multirow[t]{9}{*}{ Luminal A } & PIK3CA & p.N345K & 62 & 36 & $\mathrm{~N}$ & $2 a$ & + & + & - \\
\hline & PIK3CA & p.E545K & 46 & 36 & N & $2 a$ & + & + & - \\
\hline & PIK3CA & p.E545K & 61 & 47 & N & $2 a$ & + & + & - \\
\hline & PIK3CA & p.H1047L & 55 & 36 & N & $2 b$ & + & + & - \\
\hline & PIK3CA & p.H1047R & 51 & 48 & $\mathrm{~N}$ & $2 a$ & + & + & - \\
\hline & PIK3CA & p.H1047R & 46 & 42 & N & $2 a$ & + & + & - \\
\hline & PIK3CA & p.H1047R & 56 & 36 & N & $2 a$ & + & - & - \\
\hline & PIK3CA TP53 & p.H1047R/p.R248W & 57 & 41 & $\mathrm{~N}$ & $2 a$ & + & + & - \\
\hline & PIK3CA TP53 & p.H1047R/p.R175H & 50 & 36 & Y & $2 b$ & + & + & - \\
\hline \multirow[t]{6}{*}{ Luminal B/HER2- } & PTEN & p.T321fs*23 & 55 & 28 & Y & $3 c$ & + & + & - \\
\hline & PIK3CA TP53 & p.E545K/ p.H193R & 52 & 53 & N & $3 c$ & + & + & - \\
\hline & PIK3CAVBRAF & p.H1047R/ p.V600M & 59 & 36 & Y & $3 b$ & + & + & - \\
\hline & PIK3CA & p.H1047R & 52 & 36 & $\mathrm{~N}$ & $3 a$ & + & + & - \\
\hline & PIK3CA & p.H1047R & 58 & 36 & N & $3 a$ & + & + & - \\
\hline & PIK3CA & p.H1047R & 68 & 54 & N & $3 a$ & + & + & - \\
\hline \multirow[t]{11}{*}{ Luminal B/HER2+ } & GNAS/IDH1/KRAS & p.R201C/ p.R132C/ p.G12D & 57 & 36 & N & $2 a$ & + & + & + \\
\hline & PIK3CA & p.E542K & 50 & 47 & N & $3 a$ & + & + & + \\
\hline & PIK3CA & p.E545K & 66 & 42 & $\mathrm{~N}$ & $2 b$ & + & + & + \\
\hline & PIK3CA & p.H1047L & 72 & 40 & N & $3 a$ & + & + & ++ \\
\hline & PIK3CA & p.H1047R & 44 & 36 & N & $2 a$ & + & + & + \\
\hline & PIK3CA & p.H1047R & 45 & 55 & $\mathrm{~N}$ & $2 a$ & + & + & + \\
\hline & PIK3CA & p.H1047R & 32 & 36 & N & $2 a$ & + & - & + \\
\hline & PIK3CA & p.H1047R & 65 & 25 & Y & 3a & + & + & ++ \\
\hline & PIK3CA & p.H1047R & 63 & 12 & Y & $3 c$ & + & + & ++ \\
\hline & PIK3CAЛTP53 & p.H1047R/ p.P278L & 49 & 41 & N & $3 a$ & + & + & ++ \\
\hline & TP53 & p.Y220C & 47 & 43 & $\mathrm{~N}$ & $2 a$ & + & + & + \\
\hline \multirow[t]{4}{*}{ Triple negative } & PIK3CA & p.E545K & 60 & 43 & N & $2 b$ & - & - & - \\
\hline & PIK3CA & p.E542K & 61 & 26 & Y & 4 & - & - & - \\
\hline & TP53 & p.Y163C & 57 & 40 & N & $2 a$ & - & - & - \\
\hline & TP53 & p.R196* & 57 & 50 & N & $2 b$ & - & - & - \\
\hline \multirow[t]{2}{*}{ HER2 overexpressing } & PIK3CA & p.H1047R & 65 & 20 & Y & $3 c$ & - & - & + \\
\hline & TP53 & p.R213* & 40 & 43 & N & $2 b$ & - & - & ++ \\
\hline
\end{tabular}

DFS disease-free survival, del deletion, $f_{s}$ frameshift.

*Nonsense mutation resulting in a stop codon.

PI3K pathway [23,24]. These three mutations accounted for $88.5 \%$ of all PIK3CA mutations in our study. The remaining mutations, p.N345K and p.H1047L, are much less common and are found in less than $2 \%$ and $3.5 \%$ of breast cancers, respectively [22,25,26].

PIK3CA mutations are an early event in breast cancer development. Accordingly, we found mutations in this gene at all stages and all breast cancer subtypes in our study. While previous research has found PIK3CA mutations to be associated with older patient age [25], we did not find significant differences between age and PIK3CA mutations among all patients or breast cancer subtypes.
Consistent with a study by Kalinsky et al. [25], PIK3CA mutations were found in more HER2- tumors than HER $2+$ tumors $(61.5 \%$ vs. $38.5 \%$, respectively), although not significantly (OR: $1.20 ; P=0.81$ ). Also, patients with $\mathrm{HR}+$ tumors had a higher likelihood of having a PIK3CA mutation than those with HR- tumors, but again, this was not statistically significant (OR: $2.19 ; P=0.36)$. Others have found lower PIK3CA mutation frequencies in luminal B than luminal A breast cancers [10], but we found the opposite in our study with $53.8 \%$ of PIK3CAmutated samples as luminal B types vs. $34.6 \%$ luminal A type. Drugs like everolimus, a rapamycin derivative that 


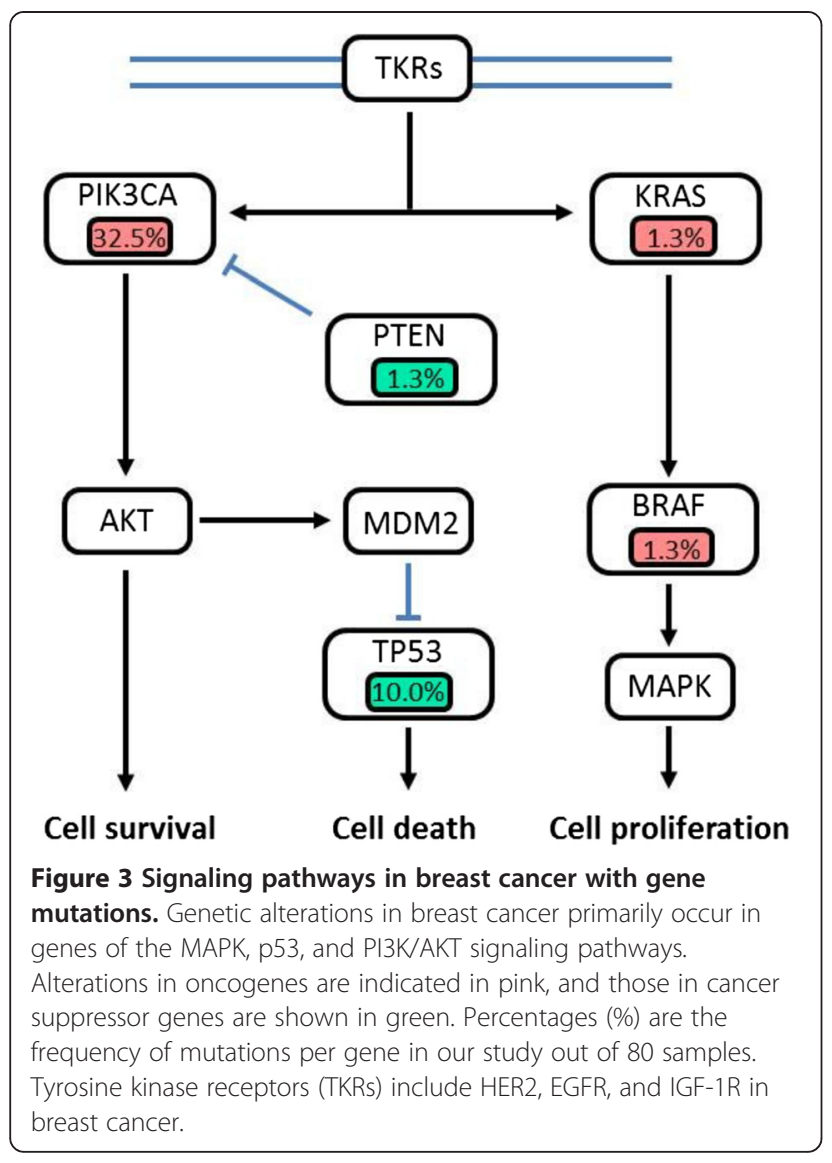

inhibits PI3K/ATK/mTOR signaling, have been approved to treat advanced HR+/HER2- breast cancer patients after other treatments have failed and have been shown to increase progression-free survival [27-29].

While the study of Kalinsky et al. found overall patient survival to be significantly improved in patients with PIK3CA mutations [25], we found that patients with PIK3CA mutations collectively had a roughly equal DFS time to patients with WT PIK3CA (38.6 vs. 38.2 months, respectively); however, patients with triple negative and HER2-overexpressing tumors and PIK3CA mutations had a shorter average DFS (34.5 and 20 months, respectively) than those with WT PIK3CA (39 and 37 moths, respectively), possibly suggesting that the other characteristics of the tumor may have greater prognostic value than PIK3CA mutations. Consistent with a clinical study on the impact of specific PIK3CA mutations on breast cancer patient prognosis [30], we found that patients with PIK3CA mutations in exon 20 had a slightly shorter DFS than those with PIK3CA mutations in exons 4 and 9 (37.4 vs. 41.3 months, respectively; $P=0.37$ ). Because activation of the PI3K/ATK/mTOR pathway has been shown to confer resistance to trastuzumab treatment in HER2+ breast tumors [31,32], and because the specific mutation may offer prognostic value, it is important to identify PIK3CA mutations in all breast cancer patients regardless of subtype.

\section{TP53 mutations}

Eight samples (10.0\%) were found to harbor TP53 mutations, and these mutations were identified in each subtype: 2 (9.5\%) in luminal A, 1 (5.9\%) in luminal B/ HER2-, 2 (8.3\%) in luminal B/HER2+, 2 (33.3\%) in triple negative, and 1 (14.3\%) in HER2-overexpressing tumors. There was no statistically significant difference between age and TP53 mutations among all patients or breast cancer subtypes, nor was there a difference in DFS and TP53 mutations and subtype. Among 80 samples, we found TP53 mutations were more likely to occur in HR- tumors (OR: $3.0 ; P=0.17$ ) and tumors of a lower grade $(75.0 \%$ at stage 2 vs. $25.0 \%$ at 3 or 4 ; OR: 2.54; $P=0.46)$. Five of the eight $(62.5 \%)$ TP53-mutated tumors were HER2-, although study wide, there was no correlation between TP53 mutations and HER2 status (OR: $1.25 ; P=1.0)$. Others have reported that TP53 is associated with worse overall patient survival [33-35], but we found this to be the opposite in our study; across all types, patients with TP53 mutations had an average DFS of 44.3 vs. 37.7 months for those with WT TP53 $(P=0.09)$.

The eight different TP53 mutations detected in our study were found at known hotspot locations, all within the DNA binding domains: two in exon 5 (p.Y136C and p.R175H); four in exon 6 (p.H193R, p.R196*, p.R213*, and p.Y220C); one in exon 7 (p.R248W); and one in exon 8 (p.P278L). Mutations in exons 5 and 7 have previously been shown to correlate with poorer overall survival and disease-free progression in breast cancer patients [36,37]. Specifically, mutations in these exons which affect the L2 and L3 loop domains of the protein (codons 163-195 and 236-251, respectively) have been found to confer resistance to certain cytotoxic drugs, including 5-fluorouracil and mitomycin [38,39]. However, in our study, there were only four patients with TP53 mutations in these exons and specific codons, and these patients had an equal DFS to patients with other TP53 mutations (44.3 months), and a longer, albeit not significant, DFS than the study average of 38.3 months $(P=0.26)$.

\section{Combination and less frequent mutations}

One advanced luminal B/HER2- sample contained a deletion in codon 321, exon 8 of PTEN, resulting in a frameshift mutation (p.T321fs*23). This mutation has been found in other cancers of the endometrium and large intestine but has not yet been identified in breast cancers [22]. As an antagonist of PI3K signaling, improper PTEN function leads to uncontrolled activation of its downstream signals [16], and reduced PTEN 
expression correlates with breast cancer progression and is associated with advanced disease [40,41]. Accordingly, the PTEN-mutated sample in our study was stage $3 C$, and the patient's DFS was 10 months shorter that the study average. Others have reported that PTEN mutations occur more often in HR- tumors $[41,42]$, which was not the case for the PTEN-mutated sample in our study.

Six of the samples (7.5\%) contained mutations in a combination of genes, and all co-mutations were found in the luminal subtypes (100\% $\mathrm{HR}+, 66.7 \%$ HER2-) (Table 3). Five of the six combination mutations cooccurred with PIK3CA, and four of these were a combination of PIK3CA and TP53. Research suggests that the presence of PIK3CA and TP53 co-mutations increase breast cancer sensitivity to some PI3K/AKT/ mTOR inhibitors when compared to those with PIK3CA mutations alone $[43,44]$; regardless, promising drugs like BEZ235, a PI3K/mTOR kinase inhibitor, may only be effective in a specific subset of triple-negative breast cancers [45].

One luminal B/HER2- sample harbored a combination mutation in PIK3CA (p.H1047R) and BRAF (p.V600M). This combination is commonly found in melanoma but rarely in breast cancer [46]. The vast majority of $B R A F$ mutations identified in all cancer types are activating mutations that occur at codon 600 , most commonly p. V600E (84.6\%), whereas p.V600M accounts for only $0.3 \%$ of $B R A F$ mutations at this codon [47]. While this mutation is not common in breast cancers, several drugs have been developed to target $B R A F$ mutations in other cancer types and are showing promising results in breast cancer models $[48,49]$. By combining treatments to target both PIK3CA and BRAF mutations, patients with combination mutations such as these may find benefit greater than a single treatment. Everolimus, however, would not be effective because research has shown this drug to be ineffective in the presence of a KRAS or BRAF mutation [27].

One stage 2A luminal B/HER2+ sample contained a unique combination of mutations in three different genes, GNAS (p.R201C), IDH1 (p.R132C), and KRAS (p.G12D), a combination that, to our knowledge, has yet to be identified in breast cancer. GNAS mutations, which are commonly found in pituitary and pancreatic cancers, and IDH1 mutations, common in gliomas, are uncommon events in breast cancers, both found in less than $1 \%[22,50]$. KRAS mutations are only slightly more common, found in $2 \%-$ $5 \%$ of breast cancers [22,51]. KRAS is involved in the EGFR signaling pathway, and anti-EGFR drugs like lapatinib used to treat advanced HER2+ breast cancers require WT KRAS to be effective $[52,53]$. For a patient with these tumor characteristics, knowledge of mutated KRAS status would prevent unnecessary drug toxicity.

\section{Conclusion}

Characterization of breast cancer tumors is critical in determining appropriate treatment options and predicting patient prognosis. The hormone receptor status and HER2 expression act as markers to direct drug treatments or to predict behavior of the disease. In addition to immunohistochemical properties, it is becoming increasingly evident that gene mutations play a role in breast cancer progression and response to treatment, making it critical to determine the genetic profile of each breast cancer tumor to personalize treatments and optimize patient outcomes. In our study, we used Ion Torrent sequencing technology to identify mutations in 80 clinical breast cancer tumors of various subtypes. Our study revealed not only uncommon and novel combination mutations but also mutations commonly found in breast cancers at frequencies similar to those previously reported, indicating the reliability of the Ion Torrent sequencing method to genotype cancer samples in a clinical setting. Ion Torrent sequencing technology has also been shown to be more cost and time effective than other traditional sequencing methods [19,54], and as such, it may be a feasible way to advance personalized patient treatments by providing clinicians a tool to characterize breast cancers beyond immunohistochemical markers and ultimately improve outcomes for breast cancer patients.

\section{Additional file}

Additional file 1: Table S1. Frequencies of missense point mutations, insertion, and deletion mutations in 737 mutational hotspot regions of 45 genes in 80 breast cancer samples.

\section{Competing interests}

Authors Chuanning Tang, Hua Ye, Feng Lou, Dandan Zhang, Hong Sun, Haichao Dong, Guangchun Zhang, Zhiyuan Liu, Zhishou Dong, Baishuai Guo, He Yan, Chaowei Yan, Lu Wang, Ziyi Su, and Yangyang Li are employees of San Valley Biotechnology, Inc. in Beijing, China.

\section{Authors' contributions}

$S L, H W, L Z, C T, H Y, L B, A W, F L, D Z, H S, H D, G Z, Z L, Z D, B G, H Y, C Y, L W, Z S$, $Y L$, and $T Z$ performed the breast cancer tumor sample sequencing, data analysis, and figure preparation. SL, HW, LZ, SYC, and TZ designed the study, performed the data analysis, and assisted with the manuscript preparation. LJ, XFH, and SYC performed the additional data analysis, wrote the main manuscript text, and prepared the manuscript tables. All authors read and approved the final manuscript.

\section{Acknowledgements}

We would like to thank Rong Shi at the Wu Jieping Foundation, Dr. Haibo Wang, Ying Li, and other members of San Valley Biotechnology Inc. Beijing for their assistance in sample and data collection. We would also like to thank the staffs at the Beijing Military Hospital for their generous support for DNA sequencing and data collection. This research was supported by the grants from the Wu Jieping Foundation and the National Institute of Health (R01 CA90427 \& R01 Al084811 to SY Chen).

\section{Author details}

${ }^{1}$ The First Affiliated Hospital of Dalian Medical University, Dalian, Liaoning, China. ${ }^{2}$ San Valley Biotechnology Incorporated, Beijing, China. ${ }^{3}$ Norris 
Comprehensive Cancer Center, Department of Molecular Microbiology and Immunology, Keck School of Medicine, University of Southern California, Los Angeles, CA, USA.

\section{Received: 28 November 2014 Accepted: 18 January 2015 Published online: 08 February 2015}

\section{References}

1. Ferlay J, Soerjomataram I, Ervik M, Dikshit R, Eser S, Mathers C, et al. GLOBOCAN 2012 v1.0, Cancer incidence and mortality worldwide: IARC CancerBase No. 11 [Internet]. Lyon, France: International Agency for Research on Cancer; 2013. Available from: http://globocan.iarc.fr, accessed on 9/10/2014.

2. Wang B, He M, Wang L, Engelgau MM, Zhao W, Wang L. Breast cancer screening among adult women in China, 2010. Prev Chronic Dis. 2013;10:E183.

3. He F-J, Chen J-Q. Consumption of soybean, soy foods, soy isoflavones and breast cancer incidence: differences between Chinese women and women in Western countries and possible mechanisms. Food Sci Hum Wellness. 2013;2:146-61.

4. Brody JG, Rudel RA, Michels KB, Moysich KB, Bernstein L, Attfield KR, et al. Environmental pollutants, diet, physical activity, body size, and breast cancer. Cancer. 2007;109:2627-34.

5. Fan L, Strasser-Weippl K, Li JJ, St Louis J, Finkelstein DM, Yu KD, et al. Breast cancer in China. Lancet Oncol. 2014;15:e279-289.

6. Parise CA, Caggiano V. Breast cancer survival defined by the ER/PR/HER2 subtypes and a surrogate classification according to tumor grade and immunohistochemical biomarkers. J Cancer Epidemiol. 2014;2014:469251.

7. Perou CM, Sorlie T, Eisen MB, van de Rijn M, Jeffrey SS, Rees CA, et al. Molecular portraits of human breast tumours. Nature. 2000;406:747-52.

8. Ciruelos Gil EM. Targeting the PI3K/AKT/mTOR pathway in estrogen receptor-positive breast cancer. Cancer Treat Rev. 2014;40:862-71.

9. Johnston SR. New strategies in estrogen receptor-positive breast cancer. Clin Cancer Res. 2010;16:1979-87.

10. Ades F, Zardavas D, Bozovic-Spasojevic I, Pugliano L, Fumagalli D, de Azambuja E, et al. Luminal B breast cancer: molecular characterization, clinical management, and future perspectives. J Clin Oncol. 2014;32:2794-803.

11. Zhang MH, Man HT, Zhao XD, Dong N, Ma SL. Estrogen receptor-positive breast cancer molecular signatures and therapeutic potentials (review). Biomed Rep. 2014;2:41-52.

12. Ignatiadis $M$, Sotiriou C. Luminal breast cancer: from biology to treatment. Nat Rev Clin Oncol. 2013;10:494-506.

13. Abramson VG, Lehmann BD, Ballinger TJ, Pietenpol JA. Subtyping of triple-negative breast cancer: implications for therapy. Cancer. 2015;121:8-16.

14. Slamon DJ, Clark GM, Wong SG, Levin WJ, Ullrich A, McGuire WL. Human breast cancer: correlation of relapse and survival with amplification of the HER-2/neu oncogene. Science. 1987;235:177-82.

15. Wooster R, Weber BL. Breast and ovarian cancer. N Engl J Med. 2003;348:2339-47.

16. Chalhoub N, Baker SJ. PTEN and the PI3-kinase pathway in cancer. Annu Rev Pathol. 2009;4:127-50.

17. Lee JW, Soung YH, Kim SY, Lee HW, Park WS, Nam SW, et al. PIK3CA gene is frequently mutated in breast carcinomas and hepatocellular carcinomas. Oncogene. 2004;24:1477-80.

18. Mukohara T. Mechanisms of resistance to anti-human epidermal growth factor receptor 2 agents in breast cancer. Cancer Sci. 2011;102:1-8.

19. Glenn TC. Field guide to next-generation DNA sequencers. Mol Ecol Resour. 2011;11:759-69.

20. Consortium GP. A map of human genome variation from population-scale sequencing. Nature. 2010;467:1061-73.

21. Server EV: NHLBI Go Exome Sequencing Project (ESP). Seattle, WA http://evs.gs.washington.edu/EVS/ 2013:[March 19 Accessed].

22. Bamford S, Dawson E, Forbes S, Clements J, Pettett R, Dogan A, et al. The COSMIC (Catalogue of Somatic Mutations in Cancer) database and website. Br J Cancer. 2004;91:355-8.

23. Mankoo PK, Sukumar S, Karchin R. PIK3CA somatic mutations in breast cancer: mechanistic insights from Langevin dynamics simulations. Proteins Struc Funct Bioinformatics. 2009;75:499-508.

24. Bachman KE, Argani P, Samuels Y, Silliman N, Ptak J, Szabo S, et al. The PIK3CA gene is mutated with high frequency in human breast cancers. Cancer Biol Ther. 2004;3:772-5.
25. Kalinsky K, Jacks LM, Heguy A, Patil S, Drobnjak M, Bhanot UK, et al. PIK3CA mutation associates with improved outcome in breast cancer. Clin Cancer Res. 2009;15:5049-59.

26. Gymnopoulos M, Elsliger M-A, Vogt PK. Rare cancer-specific mutations in PIK3CA show gain of function. Proc Natl Acad Sci. 2007;104:5569-74.

27. Di Nicolantonio F, Arena S, Tabernero J, Grosso S, Molinari F, Macarulla T, et al. Deregulation of the PI3K and KRAS signaling pathways in human cancer cells determines their response to everolimus. J Clin Invest. 2010;120:2858-66.

28. Atkins MB, Yasothan U, Kirkpatrick P. Everolimus. Nat Rev Drug Discov. 2009;8:535-6.

29. Baselga J, Campone M, Piccart M, Burris HA, Rugo HS, Sahmoud T, et al. Everolimus in postmenopausal hormone-receptor-positive advanced breast cancer. N Engl J Med. 2012;366:520-9.

30. Lai Y-L, Mau B-L, Cheng W-H, Chen H-M, Chiu H-H, Tzen C-Y. PIK3CA exon 20 mutation is independently associated with a poor prognosis in breast cancer patients. Ann Surg Oncol. 2008;15:1064-9.

31. Nahta R, Yu D, Hung M-C, Hortobagyi GN, Esteva FJ. Mechanisms of disease: understanding resistance to HER2-targeted therapy in human breast cancer. Nat Clin Prac Oncol. 2006;3:269-80.

32. Razis E, Bobos M, Kotoula V, Eleftheraki AG, Kalofonos HP, Pavlakis K, et al. Evaluation of the association of PIK3CA mutations and PTEN loss with efficacy of trastuzumab therapy in metastatic breast cancer. Breast Cancer Res Treat. 2011;128:447-56.

33. Powell B, Soong R, lacopetta B, Seshadri R, Smith DR. Prognostic significance of mutations to different structural and functional regions of the p53 gene in breast cancer. Clin Cancer Res. 2000;6:443-51.

34. Andersen TI, Holm R, Nesland JM, Heimdal KR, Ottestad L, Borresen AL. Prognostic significance of TP53 alterations in breast carcinoma. Br J Cancer. 1993;68:540-8.

35. Soong R, lacopetta BJ, Harvey JM, Sterrett GF, Dawkins HJS, Hahnel R, et al. Detection of p53 gene mutation by rapid PCR-SSCP and its association with poor survival in breast cancer. Int J Cancer. 1997;74:642-7.

36. Berns EM, van Staveren IL, Look MP, Smid M, Klijn JG, Foekens JA. Mutations in residues of TP53 that directly contact DNA predict poor outcome in human primary breast cancer. Br J Cancer. 1998;77:1130-6.

37. Cuny M, Kramar A, Courjal F, Johannsdottir V, lacopetta B, Fontaine $H$, et al. Relating genotype and phenotype in breast cancer: an analysis of the prognostic significance of amplification at eight different genes or loci and of p53 mutations. Cancer Res. 2000;60:1077-83.

38. Geisler S, Lonning PE, Aas T, Johnsen H, Fluge O, Haugen DF, et al. Influence of TP53 gene alterations and c-erbB-2 expression on the response to treatment with doxorubicin in locally advanced breast cancer. Cancer Res. 2001;61:2505-12.

39. Geisler S, Borresen-Dale AL, Johnsen H, Aas T, Geisler J, Akslen LA, et al. TP53 gene mutations predict the response to neoadjuvant treatment with 5 -fluorouracil and mitomycin in locally advanced breast cancer. Clin Cancer Res. 2003;9:5582-8.

40. Bose S, Crane A, Hibshoosh H, Mansukhani M, Sandweis L, Parsons R. Reduced expression of PTEN correlates with breast cancer progression. Hum Pathol. 2002;33:405-9.

41. Depowski PL, Rosenthal SI, Ross JS. Loss of expression of the PTEN gene protein product is associated with poor outcome in breast cancer. Mod Pathol. 2001;14:672-6.

42. Saal LH, Holm K, Maurer M, Memeo L, Su T, Wang X, et al. PIK3CA mutations correlate with hormone receptors, node metastasis, and ERBB2, and are mutually exclusive with PTEN loss in human breast carcinoma. Cancer Res. 2005;65:2554-9.

43. Kim N, He N, Kim C, Zhang F, Lu Y, Yu Q, et al. Systematic analysis of genotype-specific drug responses in cancer. Int J Cancer. 2012;131:2456-64.

44. Daemen A, Griffith OL, Heiser LM, Wang NJ, Enache OM, Sanborn Z, et al. Modeling precision treatment of breast cancer. Genome Biol. 2013;14:R110

45. Moestue SA, Dam CG, Gorad SS, Kristian A, Bofin A, Maelandsmo GM, et al Metabolic biomarkers for response to PI3K inhibition in basal-like breast cancer. Breast Cancer Res. 2013;15:R16.

46. Davies H, Bignell GR, Cox C, Stephens P, Edkins S, Clegg S, et al. Mutations of the BRAF gene in human cancer. Nature. 2002;417:949-54.

47. Ihle MA, Fassunke J, Konig K, Grunewald I, Schlaak M, Kreuzberg N, et al. Comparison of high resolution melting analysis, pyrosequencing, next generation sequencing and immunohistochemistry to conventional Sanger 
sequencing for the detection of p.V600E and non-p.V600E BRAF mutations. BMC Cancer. 2014;14:13.

48. Santarpia L, Qi Y, Stemke-Hale K, Wang B, Young EJ, Booser DJ, et al. Mutation profiling identifies numerous rare drug targets and distinct mutation patterns in different clinical subtypes of breast cancers. Breast Cancer Res Treat. 2012;134:333-43.

49. Nagaria TS, Williams JL, Leduc C, Squire JA, Greer PA, Sangrar W. Flavopiridol synergizes with sorafenib to induce cytotoxicity and potentiate antitumorigenic activity in EGFR/HER-2 and mutant RAS/RAF breast cancer model systems. Neoplasia. 2013;15:939-51.

50. Bleeker FE, Lamba S, Leenstra S, Troost D, Hulsebos T, Vandertop WP, et al. IDH1 mutations at residue p.R132 (IDH1R132) occur frequently in high-grade gliomas but not in other solid tumors. Hum Mutat. 2009;30:7-11.

51. Karnoub AE, Weinberg RA. Ras oncogenes: split personalities. Nat Rev Mol Cell Biol. 2008:9:517-31.

52. Lv N, Lin S, Xie Z, Tang J, Ge Q, Wu M, et al. Absence of evidence for epidermal growth factor receptor and human homolog of the Kirsten rat sarcoma-2 virus oncogene mutations in breast cancer. Cancer Epidemiol. 2012;36:341-6.

53. Geyer CE, Forster J, Lindquist D, Chan S, Romieu CG, Pienkowski T, et al. Lapatinib plus capecitabine for HER2-positive advanced breast cancer. N Engl J Med. 2006;355:2733-43.

54. Loman NJ, Misra RV, Dallman TJ, Constantinidou C, Gharbia SE, Wain J, et al. Performance comparison of benchtop high-throughput sequencing platforms. Nat Biotech. 2012;30:434-9.

\section{Submit your next manuscript to BioMed Central and take full advantage of:}

- Convenient online submission

- Thorough peer review

- No space constraints or color figure charges

- Immediate publication on acceptance

- Inclusion in PubMed, CAS, Scopus and Google Scholar

- Research which is freely available for redistribution 